\title{
ORIGEM E RAMIFICAÇÕES DAS ARTÉRIAS MESENTÉRICAS CRANIAL E CAUDAL EM TARTARUGA DA AMAZÔNIA Podocnemis expansa - Schweigger, 1812, (Testudinata-pelomedusidae) (Origin and ramifications of the cranial and caudal mesenteric arteries in Amazonian turtle - Podocnemis expansa Schweigger, 1812, Testudinata-Pelomedusidae)
}

\author{
SANTOS, A.L.Q. '; MUNDIM, A.V. ${ }^{1}$; MORAES, F.M. ${ }^{2}$; ALVARENGA, G.J.R. ${ }^{3}$; \\ MARQUES, F.K. ${ }^{4}$; BORGES, K.M. ${ }^{4}$; ALVES JÚNIOR, J.R.F. ${ }^{5}$
}

\begin{abstract}
${ }^{1}$ Professor Doutor da Faculdade de Medicina Veterinária (Laboratório de Pesquisa em Animais Silvestres) da Universidade Federal de Uberlândia; ${ }^{2}$ Graduando da Faculdade de Medicina Veterinária da Universidade Federal de Uberlândia, Bolsista de Iniciação Científica CNPq;

${ }^{3}$ Graduanda da Faculdade de Medicina Veterinária da Universidade Federal de Uberlândia, Bolsista de Iniciação Científica FAPEMIG; ${ }^{4}$ Graduanda da Faculdade de Medicina Veterinária da Universidade Federal de Uberlândia. ${ }^{5}$ Pós - graduando da Faculdade de Medicina Veterinária da Universidade Federal de Uberlândia.
\end{abstract}

RESUMO - Foram estudadas cinco Podocnemis expansa, fêmeas, cujo peso variava de 550 a $850 \mathrm{~g}$. Os animais tiveram seu sistema arterial injetado com solução corada de látex sintético, fixados e armazenados em solução aquosa de formaldeído a 10\%. Após abertura da cavidade celomática, as artérias mesentéricas cranial e caudal foram dissecadas. Encontrou-se a artéria mesentérica cranial como um ramo do tronco celíaco-mesentérico e originando as artérias pancreaticoduodenal caudal, ileocólica e jejunais, que irrigam o duodeno, pâncreas, cólon, íleo e jejuno, respectivamente. A artéria mesentérica caudal origina-se da aorta $(60 \%)$ ou da artéria ilíaca comum (40\%), distribuindo-se para o cólon e reto.

Palavras chave: Podocnemis expansa, Artérias, Intestino, Anatomia.

ABSTRACT - An anatomic study has been carried out on the mesenteric arteries of five Podocnemis expansa females weighing from 550 to $680 \mathrm{~g}$. The animals had their artery system injected with a latex solution colored with a specific pigment and were then fixed in a $10 \%$ formol solution. After the coelomatic cavity being opened the cranial and caudal mesenteric arteries were dissected. It has been found that the cranial mesenteric artery is a branch of the coeliac mesenteric trunk while the cranial mesenteric artery gives off the pancreatic-duodenal, ileocolic and jejunal arteries that irrigate the duodenum, pancreas, colon, ileum and jejunum respectively. The aorta gives origen of as much as $60 \%$ of the caudal mesenteric artery, the remaining $40 \%$ being originated from the common ileac artery, their branches reaching the colon and the rectum.

Key words: Podocnemis expansa, Arteries, Gut, Anatomy.

\section{Introdução}

As funções primárias do trato digestório são a digestão e absorção de nutrientes essenciais aos processos metabólicos dos animais, além de revelar hábitos alimentares gerais, sendo útil para a sistemática. Os nutrientes digeridos são absorvidos pelo epitélio e transferidos para a circulação (HILDEBRAND, 1995).

Ainda para HILDEBRAND (1995), o sistema vascular-sangüíneo dos vertebrados é denominado de sistema fechado, pois é um sistema contínuo de ductos que funciona transportando gases respiratórios, nutrientes, resíduos metabólicos, hormônios e anticorpos. Serve na manutenção do meio interno, removendo do corpo materiais tóxicos e patogênicos e funciona na regulação da temperatura. Além disso, tem a capacidade de reparar ferimentos, compensar danos e

Correspondência para: SANTOS, L.Q. - Av. Amazonas 2245, Jardim Umuarama, Uberlândia-MG, CEP $38405-302$. quagliatto@famev.ufu.br. 
responder com surpreendente versatilidade aos diferentes requisitos do momento.

Segundo MORANDINI (1968), as artérias mesentéricas são responsáveis pelo suprimento sangüíneo do intestino delgado e grosso de répteis, aves e mamíferos.

Sobre as artérias mesentéricas, HYMAN (1949), KENT JÚNIOR (1954), HYMAN (1957), MONTAGNA(1973), ZISWILLER (1980), tecem considerações, relatando que são responsáveis pela irrigação do intestino delgado e grosso.

De todos os répteis, as tartarugas talvez sejam as mais conhecidas pela população e a Tartaruga da Amazônia (Podocnemis expansa) é um dos maiores quelônios de água doce da América do Sul, embora pouco se pesquise sobre esta espécie.

A Podocnemis expansa pertence ao filo Chordata, ao subfilo Vertebrata, a superclasse Tetrapoda, à classe Reptilia e à ordem Chelonia (MORANDINI, 1968, RENNER e CUNHA, 1969, BENZZONI, 1972, GRASSÉ, 1978, ZISWILLER, 1978, ZISWILLER, 1980, ROMER e PARSONS, 1985, ORR, 1986). Apresenta um corpo inteiramente coberto por um casco (carapaça superior) e o plastrão na porção inferior, que são fortes, de onde somente a cabeça, os membros e a cauda emergem. Os dentes das tartarugas foram perdidos na escala evolutiva e as mandíbulas são cobertas por placas córneas e aguçadas (VILLE et al., 1985).

A presente pesquisa visou obter informações sobre o sistema circulatório dessa espécie animal; mais especificamente o comportamento das artérias mesentéricas cranial e caudal, afim de diminuir a escassez de informações em relação ao assunto em questão, bem como fornecer subsídios para outras áreas do conhecimento.

\section{Material e Método}

Utilizou-se cinco Podocnemis expansa (Schweigger, 1812, Testudinata, Pelomedusidae), fêmeas, com peso variando de 550 a $850 \mathrm{~g}$, procedentes do rio Araguaia, município de Cocalinhos - MT (Licença IBAMA № 013/97-DIFAS).

\section{FIGURA 1 - DESENHO ESQUEMÁTICO DAS ARTÉRIAS MESENTÉRICAS CRANIAL ) E CAUDA Podocnemis expansa. AAD: ARCO AÓRTICO DIREITO; AA: AORTA; AAE: ARCO AÓRTICO ESQUERDO; AG: ARTÉRIA GÁSTRICA; AIC: ARTÉRIA ÍLEO-CÓLICA; AIICO: ARTÉRIA ILÍACA COMUM; AJ: ARTÉRIAJEJUNAL; AL: ARTÉRIALIENAL; AMCa:ARTÉRIAMESENTÉRICACAUDAL; AMCr: ARTÉRIA MESENTÉRICA CRANIAL; APDCa: ARTÉRIA PANCREATICODUODENAL CAUDAL; APDCr: ARTÉRIAPANCREÁTICO-DUODENAL CRANIAL; RG: RAMO GÁSTRICO; TCM: TRONCO CELÍACO-MESENTÉRICO.}

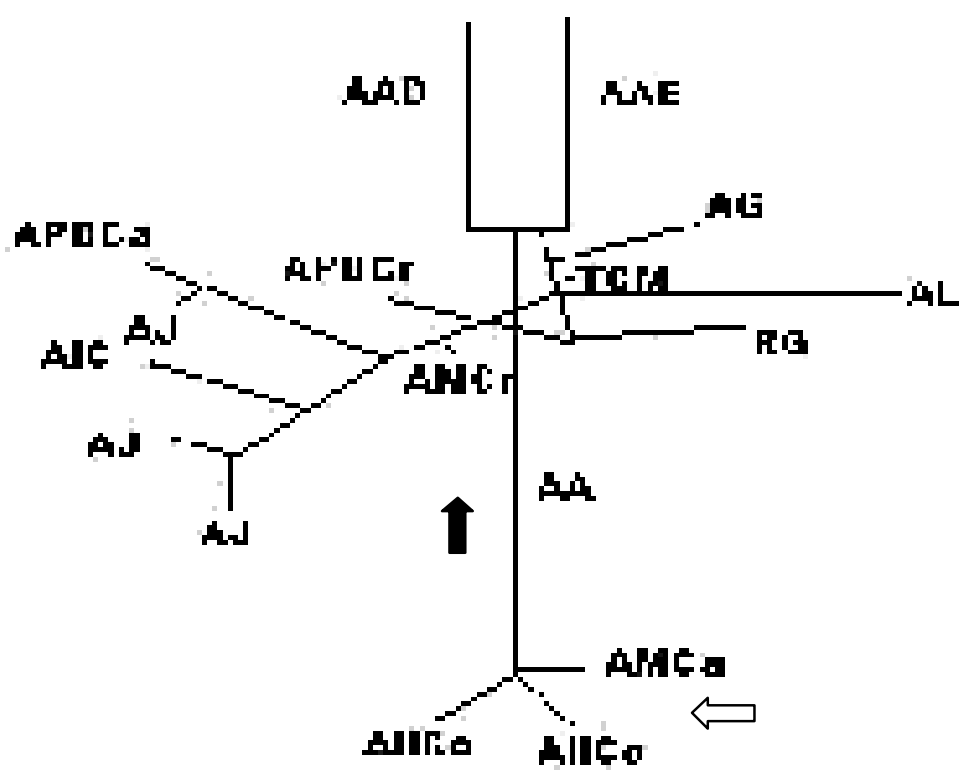


Os animais foram encaminhados ao Laboratório de Pesquisa em Animais Silvestres (LAPAS) da Faculdade de Medicina Veterinária da Universidade Federal de Uberlândia, onde, por dissecação na região cervical, individualizouse as artérias carótidas comuns. Com auxílio de cânula, de calibre compatível com o diâmetro do vaso $(2 \mathrm{~mm}$ de diâmetro por $50 \mathrm{~mm}$ de comprimento), procedeu-se a "lavagem", ou seja, a desobstrução do sistema vascular com solução fisiológica e, em seguida, injetou-se solução de Neoprene Látex "450" (Du Pont do Brasil S.A. - Indústrias Químicas) corada com pigmento específico (Globo S.A.-Tintas e Pigmentos). Logo após, o material foi fixado em solução aquosa de formaldeído a $10 \%$, por período mínimo de 96 horas, antes que se iniciasse a dissecação.

FIGURA 2 - DESENHO ESQUEMÁTICO DAS ARTÉRIAS MESENTÉRICAS NA Podocnemis expansa, MOSTRANDO A ARTÉRIA MESENTÉRICA CAUDAL (AMCa) ORIGINANDO-SE DA ARTÉRIA ILÍACA COMUM ( 介 )AAD: ARCO AÓRTICO DIREITO; AA: AORTA; AAE: ARCO AÓRTICO ESQUERDO; AG: ARTÉRIA GÁSTRICA; AHE: ARTÉRIA HEPÁTICA ESQUERDA; AIC: ARTÉRIA ÍLEO-CÓLICA; AIICO: ARTÉRIA ILÍACA COMUM; AJ: ARTÉRIA JEJUNAL; ALARTÉRIA LIENAL; AMCr: ARTÉRIA MESENTÉRICA CRANIAL; APDCa: ARTÉRIA PANCREATICODUODENAL CAUDAL; APDCr:ARTÉRIA PANCREÁTICODUODENAL CRANIAL; RG: RAMO GÁSTRICO; TCM: TRONCO CELÍACO-MESENTÉRICO.

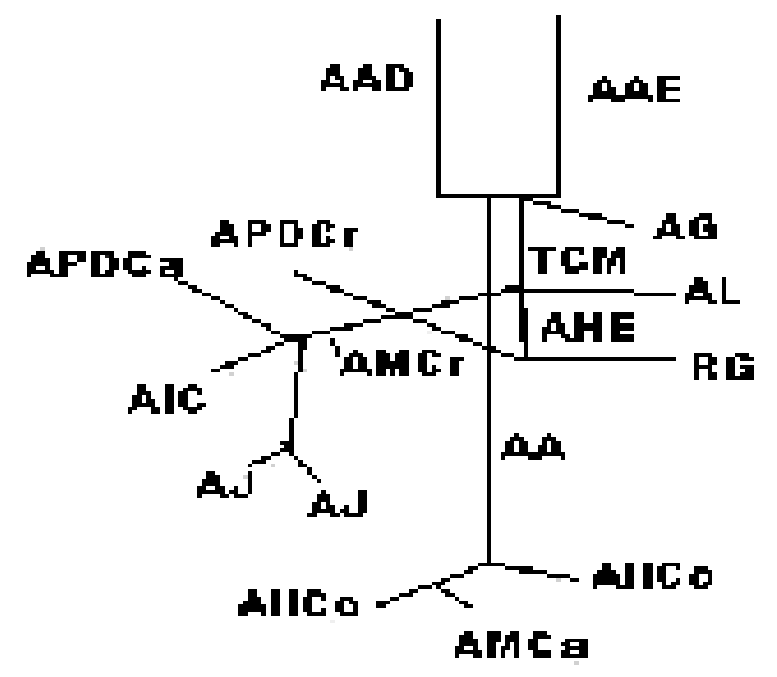

Após abertura da cavidade celomática, foram localizadas e dissecadas as artérias mesentéricas cranial e caudal, registrando-se e confeccionando-se esquemas de suas origens e ramificações (FIGURAS 1 e 2 ).

\section{Resultados e Discussão}

MORANDINI (1968), RENER E CUNHA (1969), BENZZONI (1972), GRASSÉ (1978), ZISWILLER (1978), ZISWILLER (1980), PINSETA et al. (1984-85), ORR (1986), AMABIS e MARTHO (1990), POUGH et al. (1993), HILDEBRAND (1995), STORER et al. (1995) e SOARES (1998), fornecem apenas informações genéricas sobre a biologia dos répteis (classificação biológica, habitat, hábito alimentar, respiração, coração) e algumas considerações sobre a disposição dos vasos sangüíneos destes animais.

Nos animais dissecados, observou-se que a artéria mesentérica cranial (FIGURA 1) surge como um ramo do tronco celíaco-mesentérico, em posição ventrolateral esquerda, e dá origem às artérias pancreaticoduodenal caudal, ileocólica e jejunais. Essa observação coincide com as descrições de WALKER JÚNIOR (1970), quando cita que as artérias mesentéricas são um ramo da artéria celiacomesentérica, e com MONTAGNA (1973), quando afirmam que a aorta envia três grandes artérias viscerais ímpares ao tubo digestivo, sendo uma delas a mesentérica superior, que se dirige ao intestino delgado por debaixo do duodeno e a parte superior do intestino delgado. 
No entanto, NOBLE e NOBLE (1940) HYMAN (1949), HYMAN (1957) e ASHLEY (1955) relatam que a artéria mesentérica superior é o terceiro ramo da artéria aorta esquerda e irriga o intestino delgado, enquanto BRELAND (1953) afirma que a artéria mesentérica superior origina-se do arco sistêmico e irriga o intestino. KENT JÚNIOR (1954) somente cita que o suprimento arterial do intestino delgado é feito através da artéria mesentérica superior. ZISWILLER (1980) faz um comentário geral, afirmando que a artéria mesentérica origina-se do arco aórtico esquerdo e irriga o intestino delgado posterior, o reto e a cloaca. FARIA (2000) e FARIA e MARIANA (2001), estudando Geochelone carbonaria, descrevem que a artéria mesentérica superior é emitida pela artéria celíaca, sendo responsável pela nutrição do mesentério e parte do intestino grosso.

$\mathrm{Na}$ Podocnemis expansa, encontrou-se a artéria pancreaticoduodenal caudal fornecendo ramos para o duodeno e pâncreas e ainda, em dois animais (40\%), emitindo uma artéria jejunal, que fornece ramos para o jejuno e íleo. Em todos os animais ocorreu a anastomose com a artéria pancreaticoduodenal cranial, dando origem à artéria hepática direita.

A artéria ileocólica distribuiu-se no cólon e, ainda, em três casos (60\%), no íleo; e em 2 casos (40\%) no jejuno. Em dois animais (40\%) houve a anastomose da artéria ileocólica com a artéria mesentérica caudal.

As artérias jejunais supriram o mesentério e irrigaram o jejuno e o íleo. Em dois animais (40\%) observou-se a anastomose das artérias jejunais com um ramo da artéria ileocólica, em dois animais $(40 \%)$ com um ramo da artéria pancreaticoduodenal caudal e, em um animal $(20 \%)$, com a artéria jejunal, originada da artéria ilíaca comum.

A artéria mesentérica caudal, na Podocnemis expansa, teve origem da aorta em 3 animais (60\%), assim como citam HYMAN (1957) e MONTAGNA (1973), e da artéria ilíaca comum, em 2 casos (40\%) (FIGURA 2), distribuindo-se no cólon e no reto, considerando-se que o vaso sangüíneo denominado de aorta dorsal por estes autores corresponde à aorta deste estudo, conforme recomenda o I.C.V.G.A.N. (1994).
Em dois animais (40\%) observou-se a anastomose da artéria mesentérica caudal com um ramo da artéria ileocólica.

Ainda sobre a artéria mesentérica caudal, HYMAN (1949) relata apenas que a artéria mesentérica inferior irriga o intestino grosso e a cloaca. Já KENT JÚNIOR (1954) acrescenta que o suprimento arterial do intestino grosso, e glândula retal é feito através da artéria mesentérica inferior. No entanto, ASHLEY (1955) comenta sobre a artéria mesentérica posterior como sendo um dos ramos da mesentérica anterior, continuando-se no intestino grosso acompanhando todo o caminho até a cloaca e, às vezes, anastomosando-se com uma das artérias ilíacas comuns. MORANDINI (1968) somente cita que a mesentérica inferior irriga o intestino grosso.

FARIA (2000) e FARIA e MARIANA (2001) comentam que a artéria mesentérica inferior é uma continuação da artéria celíaca, sendo responsável pelo abastecimento sangüíneo da região do intestino grosso e mesentério, diferindo das informações obtidas na presente pesquisa, com Podocnemis expansa, adaptando-se a denominação de mesentérica inferior para mesentérica caudal (I.C.V.G.A.N, 1994).

\section{Conclusões}

A artéria mesentérica cranial é um ramo do tronco celíaco-mesentérico e origina as artérias pancreaticoduodenal caudal, ileocólica e jejunais.

A artéria pancreaticoduodenal caudal irriga o duodeno, o pâncreas, o jejuno e o íleo e anastomosa-se com a artéria pancreaticoduodenal cranial, dando origem à artéria hepática direita.

A artéria ileocólica irriga o cólon, o íleo e o jejuno e anastomosa-se com a artéria mesentérica caudal.

As artérias jejunais irrigam o mesentério, o jejuno e íleo, e anastomosam-se com uma artéria ileocólica, ou com a artéria pancreaticoduodenal caudal, ou com a artéria ilíaca comum.

A artéria mesentérica caudal origina-se da aorta $(60 \%)$ ou da artéria ilíaca comum (40\%), distribuindo-se no cólon e reto, e anastomosase com a artéria ileocólica. 
Origem e ramificações das artérias mesentéricas cranial e caudal em tartaruga da Amazônia Podocnemis expansa...

\section{Referências}

AMABIS, J.M.; MARTHO, G.R. Curso básico de biologia - seres vivos. São Paulo: Moderna, 1990. v.2, p.14-16, 338.

ASHLEY, L.M. Laboratory anatomy of the turtle. Dubuque: WM. C. Brown, 1955. p.26-34.

BENZZONI, P. Compêndio de zoologia. Belo Horizonte: Livraria Cultural Brasileira, 1972. p.131162.

BRELAND, O.P. Manual of comparative anatomy. 2.ed. New York: McGraw-Hill Book Company, 1953. p.122-124.

FARIA, T.N. Descrição da origem, trajeto e número das principais artérias do jabuti "Geochelone carbonaria" (Spix, 1824). São Paulo, 2000. 58 f. Dissertação (Mestrado em Medicina Veterinária) - Faculdade de Medicina Veterinária e Zootecnia, Universidade de São Paulo.

FARIA, T.N.; MARIANA, A.N.B. Origens e ramificações das artérias aortas esquerda e dorsal do jabuti (Geochelone carbonaria, Spix, 1824). Brazilian Journal of Veterinary Research and Animal Science, São Paulo, v.38, n.4. p.155-159, 2001.

GRASSÉ, P.P. Zoologia: vertebrados reproducción, biologia, evolucion y sistemática agnatos, peces, anfibios y reptiles. Barcelona: Toray-Masson, 1978. v.3, p.1-9.

HILDEBRAND, M. Análise da Estrutura dos vertebrados. São Paulo: Atheneu, 1995. p.263.

HYMAN, L.H. Comparative vertebrate anatomy. Chicago: University of Chicago, 1949. p.346.

HYMAN, L.H. Comparative vertebrate anatomy. Ilinóis: The University of Chicago Press, 1957. p.344-8.

INTERNATIONAL COMMITTEE ON VETERINARY GROSS ANATOMICAL NOMENCLATURE I.C.V.G.A.N. Nomina anatomica veterinaria. 4. ed. New York, 1994. (Together with nomina histologica, 2 ed, 1994 and nomina embriologica veterinary, 1994).
KENT JÚNIOR, G.C. Comparative anatomy of the vertebrates. New York: McGraw-Hill Book Company, 1954. p.377-379.

MONTAGNA, W. Anatomia comparada. 2. ed. Barcelona: Omega, 1973. p.212.

MORANDINI. C. Zoologia. 2. ed. São Paulo: Nobel, 1968. v.4, p.1196.

NOBLE, G.A.; NOBLE, E.R. Anatomy of the turtle. Califórnia: Stanford University Press, 1940. p.1-18.

ORR, R.T. Biologia dos vertebrados. 5. ed. São Paulo: Roca, 1986. p.100-102.

PINSETA, D.E.; GUASELLI FILHO, E.; SASSON, S. Biologia - fisiologia animal comparada. São Paulo: Anglo, 1984-85. p.37, 92-93. (Coleção Anglo. Biologia, 43).

POUGH, F.H.; HEISER, J.B.; MCFARLAND, W.N. A vida dos vertebrados. São Paulo: Atheneu, 1993. p.386- 409.

RENNER, M.; CUNHA, A.X. Guia de trabalhos práticos de zoologia. 15. ed. Coimbra: Atlântica, 1969. p.341-415.

ROMER, A.S.; PARSONS, T.S. Anatomia comparada dos vertebrados. São Paulo: Atheneu, 1985. p.381.

SOARES, J.L. Fundamentos de biologia dos seres vivos: estrutura e funções. São Paulo: Scipione, 1998. v.2, p.332.

STORER, T.I.; USINGER, R.L.; STEBBINS, R.C.; NYBAKKEN, J.W. Zoologia geral. 6. ed. São Paulo: Nacional, 1995. p.267-289, 642-667.

VILLE, C.A.; WALKER Jr, N.F.; BARNES, R.D. Zoologia geral. 6. ed. Rio de Janeiro: Editora Interamericana, 1985. p.548.

WALKER JÚNIOR, W. Vertebrate dissection. 4.ed. Philadelphia: W.B. Saunders, 1970. p.210-311.

ZISWILLER, V. Zoologia especial dos vertebrados. Barcelona: Omega, 1978. v.1, p.1-3.

ZISWILLER, V. Zoologia especial dos vertebrados. Barcelona: Omega, 1980. v.2, p.100101.

Recebido para publicação: 22/03/2004

Aprovado:

$20 / 06 / 2004$ 